\title{
A potato model built using the APSIM Plant.NET Framework
}

\author{
$\underline{\text { H.E. Brown }}^{\text {a }}$, N. Huth ${ }^{\text {b }}$ and D. Holzworth ${ }^{b}$ \\ ${ }^{a}$ The New Zealand Institute for Plant and Food Research, Private Bag 4704, Christchurch, New Zealand \\ ${ }^{b}$ CSIRO Ecosystem Sciences, 203 Tor St, Toowoomba, Queensland 4350, Australia. \\ Email: Hamish.brown@plantandfood.co.nz
}

\begin{abstract}
The APSIM potato model is a comprehensive daily time step, deterministic potato crop model build in the APSIM Plant.net Framework which integrates with the APSIM soil, management and user interface components to provide a robust and user friendly potato crop model. This paper provides a brief description of the potato models mechanisms with a particular focus on the arbitrator module that was developed to enable explicit linkages between the partitioning of dry matter (DM) and nitrogen $(\mathrm{N})$.
\end{abstract}

Potential DM production is estimated from the product of solar radiation, radiation interception, radiation use efficiency and stress factors (water, temperature and $\mathrm{CO}_{2}$ ). Radiation interception is calculated from predictions of leaf area index (LAI) and an extinction coefficient. The estimation of LAI is created using a phytomer type canopy model that uses inputs of tuber planting density and the number of stems per tuber to determine the population density of primary stem units. It then predicts the appearance, expansion, size and duration of individual leaves on primary stems and the occurrence of branching to give a canopy model that can explicitly account for the effects of planting arrangement and seed tuber characteristics on the development of the crops canopy.

One of the most important elements of Plant.NET models is the arbitrator which determines how much of potential DM production can be assimilated and the partitioning of DM and $\mathrm{N}$ between competing organs. An alternative to the existing arbitrator module was written for the potato model, based on the concepts of partitioning used in the SIRIUS wheat model. This arbitrator may be used for other crop models built in Plant.NET. The new arbitrator gets each organs DM supplies and demands and then allocates potential DM accordingly. If DM supply is greater than demand it will partition excess to sink organs and if DM supply is less than demand it will remobilize non-structural DM from organs that have labile DM components. Potential DM allocations and accumulated $\mathrm{N}$ deficit are used to calculate each organs $\mathrm{N}$ demand and then the arbitrator steps through a series of $\mathrm{N}$ allocation routines. The first is the reallocation of $\mathrm{N}$ from senescing organs, followed by the allocation of $\mathrm{N}$ available for uptake from the soil and the last is the remobilization of $\mathrm{N}$ from non-structural pools in organs. Once $\mathrm{N}$ allocation is set the arbitrator then determines if this is enough to assimilate the potential DM allocation and if not DM allocation is reduced so each organ maintains it minimum $\mathrm{N}$ concentration.

Testing and parameterization is ongoing but with current progress the model has been tested against a number of datasets and has performed well. The most extensive testing has occurred on $\mathrm{N}$ uptake and response. The model gives realistic reproduction of the effects of $\mathrm{N}$ shortage on both tuber yield and the time course of tuber $\mathrm{DM}$ accumulation. In particular it predicts substantial plasticity in the $\mathrm{N}$ concentration of tubers such that large differences in tuber $\mathrm{N}$ uptake have little impact on tuber growth. It predicts that stem growth will be the first thing to stop under $\mathrm{N}$ shortage and the plant will draw down stem $\mathrm{N}$ concentrations to maintain leaf area and tuber growth. Leaf growth is next to be reduced to ensure tuber $\mathrm{N}$ supply is maintained and finally tuber growth will slow if $\mathrm{N}$ shortage persists long enough for tubers to fall to their minimum $\mathrm{N}$ concentration.

Having a comprehensive potato model operating in the APSIM platform will be valuable to a wide range of model users interested in the physiology and agronomy of potato. The model is currently available to advanced users and will be available in general release, once all release criteria have been satisfied.

Keywords: canopy, dry matter, nitrogen, partitioning, yield. 


\section{INTRODUCTION}

Potato is the $4^{\text {rd }}$ most important food crop in the world behind rice, wheat and maize and its global production has increased substantially over the last 20 years (http://www.potato2008.org/en/world/index.html). Potato is the subject of substantial research and development efforts (Struik et al., 2007) and has been the first of the 4 major staple food crops to have its genome map completed. However, the attention that potato crop physiology has received and the subsequent state of development of potato crop models has lagged well behind that of wheat, maize and rice. A search on the Web of Knowledge for physiological research that may contribute to the development of potato crop models (using the search expression "potato and (radiation interception or radiation use efficiency or photosynthesis or extinction coefficient or phyllochron or leaf appearance or leaf size or leaf area index or harvest index)") returns 3,324 hits. The same search with wheat, maize or rice in place of potato returns 18,697, 12,652 and 10,721 hits respectively. The reason potato physiological research and crop modeling has received so much less attention than other staple crops is not clear but it may have to do with the difficulties in quantifying the physiology of a vegetative propagated crop or the effort involved in observing the growth and development of below ground yield components. Regardless of the reason, crop models are invaluable tools for structuring current understanding of how environment, management and genotype interact to create outcomes of yield, quality and resource consumption (Haverkort, 2007). Crop models are also valuable tools in the application of research results, helping to extrapolate results beyond the confines of site and season specific trials and enabling quantitative answers to hypothetical questions. International potato research could benefit greatly from a comprehensive potato model.

This paper outlines the initial steps that have been taken to develop such a model within the APSIM modeling platform (Keating et al., 2003). APSIM was an obvious candidate for the production of such a model as it already contained robust soil water and $\mathrm{N}$ models and infrastructure for creating crop rotations and imposing management events. It also offered the Plant.NET framework (Holzworth and Huth, 2009; Huth et al., 2009). Plant.NET is a collection of classes representing plant organs, processes and functions with an interface that controls communications between classes and integrates with other APSIM components. A range of classes allow organs and processes to be represented in different levels of complexity depending on the performance needs of the specific plant model. It also provides an integrated design environment for selecting and configuring the organs, process and functions required, enabling simple yet flexible creation and enhancement of models for any plant type. The parameter values reported in this paper are based on analysis of data from a number of intensively monitored trials but there is insufficient space to present all of these analyses.

\section{MODEL STRUCTURE}

\subsection{Overview.}

This model predicts the yield, $\mathrm{N}$ uptake and water use of potato crops within the APSIM model framework (Keating et al., 2003). Descriptions of the soil specific aspects of water and $\mathrm{N}$ balance are well covered by (Keating et al., 2003) and many others so this paper will focus on describing aspects that are specific to the potato crop model. Predictions of yield are made daily based on the production of total dry matter (DM) and its partitioning and reallocation to plant organs. Total daily DM production is calculated as in all other APSIM modules (Keating et al., 2003; Wang et al., 2004) as:

$$
\triangle D M-R a \ln \times{ }^{l} / F_{\odot} \times R U E \times f W \times f t \times f c O_{2}
$$

Where Radn is the daily solar radiation level $\left(\mathrm{MJm}^{-2}\right), I / I_{o}$ is the fraction of radiation that the crop intercepts each day. This is calculated from crop cover, the proportion of the ground that the crops canopy is covering which is calculating using an extinction coefficient of 0.8. RUE is the potential radiation use efficiency of the crop which is a constant value of $1.44 \mathrm{~g} / \mathrm{MJ}$ and RUE is multiplied by adjustment factors to account for the effects of water $(f w)$ and temperature $(f t)$ stresses and atmospheric carbon dioxide concentration $\left(f \mathrm{CO}_{2}\right)$. The value of $f w$ ranges from 1 when water is in adequate supply to 0 when the soil is completely dry. $f t$ increases from 0 at a mean daily air temperature of $2^{\circ} \mathrm{C}$ to 1.0 at a temperature of $12^{\circ} \mathrm{C}$, remains at 1 up to a temperature of $24^{\circ} \mathrm{C}$ and then declines to zero at a value of $34^{\circ} \mathrm{C}$. $f \mathrm{Co}_{2}$ has a value of 1.0 when atmospheric $\mathrm{CO}_{2}$ concentration is $350 \mathrm{ppm}$ and increases with increasing $\mathrm{CO}_{2}$ concentration as described by Ludwig and Asseng (2006). This calculation sets up potential DM production in the absence of nutrient stress. If there is insufficient $\mathrm{N}$ available for organs to achieve a minimum $\mathrm{N}$ concentration then $\mathrm{DM}$ production will be decreased below this level. This mechanism is described in detail in Section 2.4. Parameter values for extinction coefficient, RUE and stress factors for RUE responses are determined from analysis of unpublished data. 
Nitrogen uptake and water use are both limited to the minimum of the potential uptake that the fine roots can achieve and the demand from the other plant organs. Nitrogen potential uptake supply is determined from the depth of the roots, the amount of mineral $\mathrm{N}$ and the soil water content in the layers of soil that roots are present in and the crops overall root length density. For water the potential uptake is also dependent on the depth of roots. For every layer with roots present the potential uptake is calculated as the product of the available water content and an extraction rate constant. This is summed over the soil profile to give potential water extraction. Transpiration demand is calculated using the Penman-Monteith potential transpiration calculation (Snow and Huth, 2004) depending on the leaf area index and height of the crop as well as atmospheric conditions. Water stress is represented in the model as the ratio of the supply of water from the root system relative to the demand from the atmosphere. Crop processes such as leaf area expansion and $f w$ are calculated in relation to this ratio.

\subsection{Phenological development}

The duration of crop growth and changes in the crops growth behavior are dependent on the developmental status of the crop. The development of the potato crop is characterized by passage through a number of phenological stages:

1. Dormant. This phase begins when the seed tuber becomes dormant on the mother plant (some time following haulm killing) and ends when the tubers dormancy requirements are satisfied. In the current implementation of the model this is assumed to end $70{ }^{\circ} \mathrm{Cd}$ following planting but future refinements will model this explicitly, allowing dormancy to break any time before or after planting.

2. Sprouting. This phase begins with the growth of sprouts and finishes with the emergence of the shoots. The duration of this phase is dependent on the depth that seed tubers are sown, the rate of stem extension $\left(1.35 \mathrm{~mm} /{ }^{\circ} \mathrm{Cd}\right)$ and soil temperature.

3. Vegetative. In this phase the potato crop is growing only stem and leaf organs and proceeds for $300^{\circ} \mathrm{Cd}$.

4. Early Tuber. This phase begins with tuber initiation and continues until the appearance of the final primary stem leaf. During this phase the potato crop is growing leaves, stems and tubers.

5. Late Tuber. This phase goes from the appearance of the final leaf until the death of the final leaf. In this phase the potato crop only grows tuber DM.

6. Senesced. During this phase the tops of the tubers are completely senesced and the tubers lie dormant below the soil surface.

\subsection{Canopy Structure}

The structure of the canopy is modeled based on main-stem units with the number of stems initialized at planting using row spacing, seed tuber spacing within rows and the number of stems that each tuber will produce. The number of stems is currently a constant, but future developments will enable this to be modeled as a function of tuber size and treatment prior to planting. The number and size of leaves on each of the primary stem is initialized on the day of emergence. Following emergence the appearance of new leaves on the primarystem is predicted from accumulated thermal time and a phyllochron. It should be noted that (for Russet Burbank) the main-stem terminates with flowering at $\sim 16$ leaves but potato is indeterminant and branches below the terminal main-stem node continue to produce leaves after flowering (Vos, 1995). To capture this simply in the leaf model primary-stem leaves were considered to be main-stem leaves prior to flowering and the most dominant apical branch growing off the main-stem thereafter. The phyllochron increased from a value of 5 for the first leaf increasing to 30 for leaves $5-25$ and then increasing to 60 for leaves $25-34$. Phyllochron values were determined from unpublished leaf appearance measurements. The maximum leaf number was set at 34 and leaf appearance stops when this many primary stem nodes had appeared. In reality maximum leaf number will be a function of photoperiod but as all the experimental data sets being worked with to date were planted within a narrow window in spring there has been no need to implement this response.

Once leaves have appeared their potential size is calculated daily from parameters that describe:

1. Duration of the expansion period when the green area of the individual leaf is increasing. This increases from $200^{\circ} \mathrm{Cd}$ for the first leaf to $400^{\circ} \mathrm{Cd}$ at leaf 10 and remains at $400^{\circ} \mathrm{Cd}$ for all subsequent leaves.

2. Maximum size that leaves will reach if the crop is not stressed during expansion. This increases from a value of $3000 \mathrm{~mm}^{2}$ for the first leaf to $50000 \mathrm{~mm}^{2}$ for leaf 10 . It then remains at $50000 \mathrm{~mm}^{2}$ until leaf 17 and then decreases to $100 \mathrm{~mm}^{2}$ at leaf 34 .

3. Duration of a lag period that leaves will remain at maximum area. This is a constant value of $150^{\circ} \mathrm{Cd}$.

4. Duration of the senescence period during which green area of the individual leaf is contracting. This is a constant value of $100^{\circ} \mathrm{Cd}$. 
Potential leaf area expansion and specific leaf area drives leaf DM demand. If DM demand is not fully satisfied leaves will get thinner and if DM allocation is insufficient to attain the maximum specific leaf area leaf area expansion will be constrained. Leaves will also not reach their maximum size if the crop encounters water stress. The area of individual leaves may begin to decrease before the end of the lag period if the leaf is shaded by the overlying canopy. This occurs if the cover of the overlying leaf area is greater than 0.9 and $12 \%$ of the green area will senesce each day the overlying cover exceeds this threshold.

In addition to primary-stem leaves there is also extensive branching in the potato crop and the extent of secondary-stem growth is dependent on the sowing density and the number of stems per plant. Secondary-stem growth is modeled by enabling multiples of each primary-stem leaf cohort. For primary-stem leaves $1-12$ no secondary stems will occur. The potential secondary-stem production then increases to 1.0 for leaves $13-16$ and then decreases to 0 for the remainder of primary-stem nodes. This means each primary-stem can potentially produce 4 secondary stems. The timing of the appearance of these secondary-stems coincides with the appearance of the $13^{\text {th }}-16^{\text {th }}$ primary-stem leaves but their position on the main stem could be basal or apical (position of the branch is not modeled explicitly). The appearance of secondary-stem leaves occurs at the same rate as primary-stem leaves and the leaves on secondary-stems take on the same properties as the primary-stem leaves that appear at the same time. The branching pattern is based on analysis of unpublished data. The actual number of secondary stems that are initiated is dependent on crop cover at the time of potential initiation (when primary stem leaves $13-16$ are appearing). The actual number of secondary stems that are initiated is calculated as the product of the potential branch number (1.0) and the canopy cover present at the time of initiation. Secondary stem number will be 1 for covers below $50 \%$ and will decrease to zero at a cover of $80 \%$. This way if potatoes are sown at a high population or have a lot of stems per tuber, cover will have increased to a level that reduces branching. Alternatively, if a low stem population is present, the potato crop will branch extensively to ensure full ground cover.

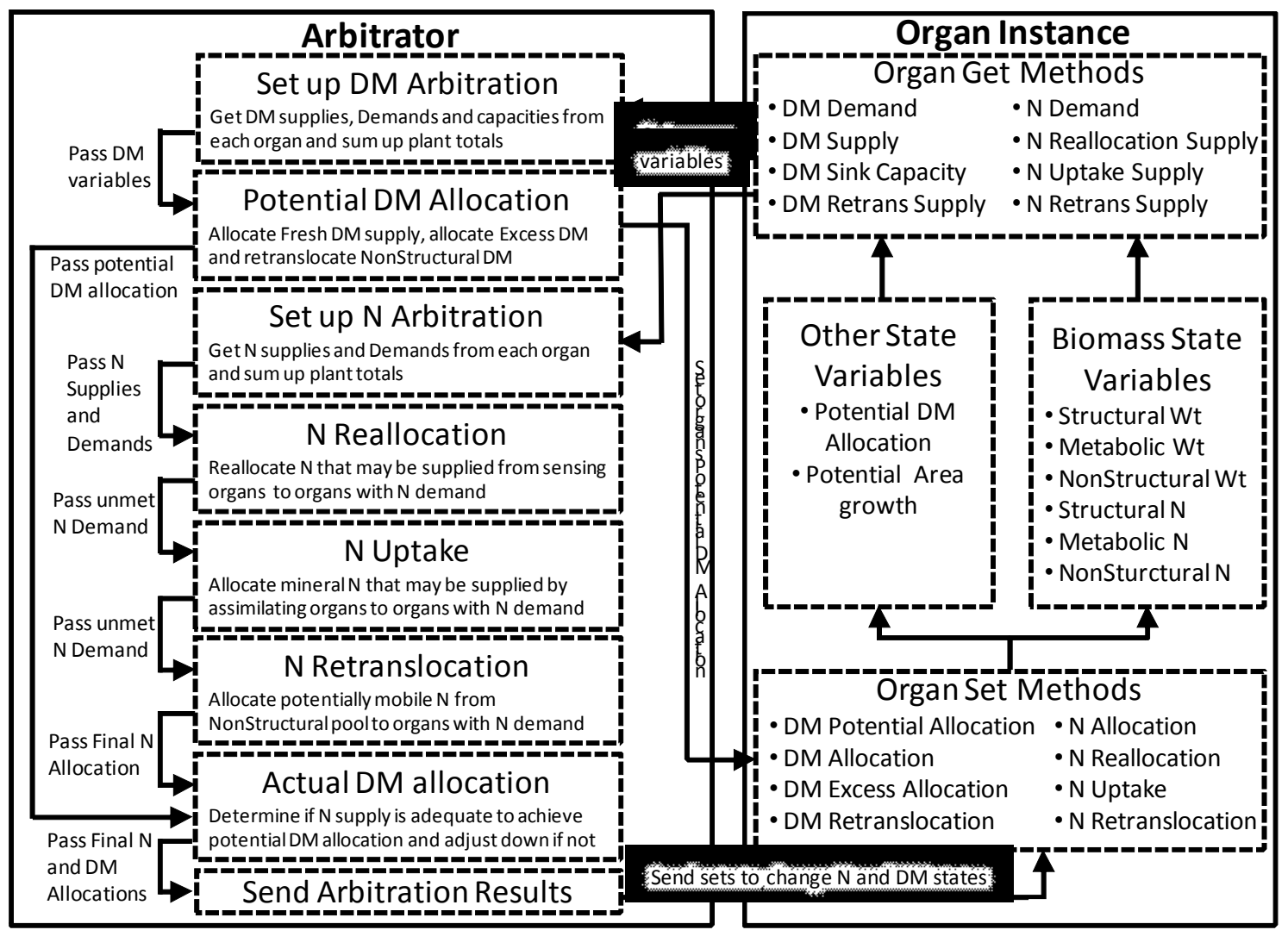

Figure 1. Schematic of the Structure of Base Organ Object, its interface with the Arbitrator Object and the processes within each time step for arbitration.

\subsection{Dry matter and Nitrogen Partitioning.}

The partitioning of DM and $\mathrm{N}$ is inherently linked within this model such that the amount of DM partitioned to an organ sets its $\mathrm{N}$ demand and the amount of $\mathrm{N}$ partitioned to an organ determines its ability to assimilate the DM that has potentially been partitioned to it. DM and $\mathrm{N}$ partitioning is implemented by an arbitrator which communicates with each organ through a standard interface (Figure 1). All organs inherit their structure from a 
base organ which has a number of get methods to communicate the organs $\mathrm{N}$ and DM supplies and demands to the Arbitrator and a number of methods where the arbitrator invokes a change in each organs DM and N status depending on the results of arbitration. Organs also use a common DM class which keeps track of the DM and $\mathrm{N}$ status of organs. Each organ may have DM and $\mathrm{N}$ in Structural, Metabolic and Non-Structural pools depending on how DM is assigned with each organs set routines. These pools may then be treated differently by other processes in the model. While there were a set of standard organs and an annual plant arbitrator in the Plant.NET framework, an alternative arbitrator and set of standard organ classes where developed for the potato model that were based on the partitioning concepts of the SIRIUS modeling approach (Jamieson et al., 1998), an approach that has been used for previous potato model development (Jamieson et al., 2004).

The arbitrator runs through a series of sequential steps (Figure 1) to determine how much DM and $\mathrm{N}$ will be allocated to each organ.

1. Firstly it sets up DM arbitration calculations by getting DM supplies and demands from each organ instance and summing supplies and demands from each organ to give plant supply and demand totals.

2. Then it does potential DM allocation. This determines how much DM each organ will get if there is sufficient $\mathrm{N}$ for the organ to reach its minimum $\mathrm{N}$ concentration. There are three steps:

a. Firstly DM Supply (freshly fixed DM from photosynthetic organs) is partitioned to organs based on their demands relative to that of the total plant demand such that if there is not enough freshly fixed DM to meet these demands the organs with the highest demands get the greatest share of the DM Supply. The demand of each organ for DM depends on their potential growth for the day. For example leaf DM depends on potential leaf area expansion and specific leaf area.

b. Next any DM Supply that was surplus to the organs DM demands is partitioned to sink organs as DM Excess Supply. An organ will be a sink if it is parameterized to have a Non-structural (mobile) component and the capacity of each organ to receive excess DM depends on its structural fraction (which determines the Non-structural Fraction and subsequent sink capacity). If sink organs are full to their capacity and some DM Supply is still unallocated this remains unallocated with the assumption that in this case the plant would down regulate photosynthesis due to lack of sink capacity.

c. Lastly, if the total plant DM Demand is not met by DM Supply the arbitrator will retranslocate Non-structural DM from Non-Structural pools (assigning a value to DM Retranslocation for each organ) to meet the DM demand of growing organs.

Once these steps are complete the arbitrator sets the DM potential allocation in each organ and moves onto $\mathrm{N}$ arbitration.

3. Firstly it sets up $\mathbf{N}$ arbitration by getting organs $\mathrm{N}$ supplies and demands and summing up totals.

Each of the following 3 steps have a number of processes in common. Firstly the arbitrator determines each organs unmet $\mathrm{N}$ demand, then it allocates $\mathrm{N}$ to each demanding organ in two passes. On the first pass the arbitrator steps through organs in order of priority, allocating $\mathrm{N}$ to meet minimum $\mathrm{N}$ demand (structural and metabolic $\mathrm{N}$ demand) to the highest priority organ before allocating any $\mathrm{N}$ to the next. For potato the order of priority is tuber, root, leaf then stem. On the second pass, $\mathrm{N}$ is allocated to meet non-structural (luxury) $\mathrm{N}$ demand relative to the size of the organs luxury $\mathrm{N}$ demand such that the organ with the greatest $\mathrm{N}$ demand will get the greatest share of the $\mathrm{N}$ supply. Finally it sets for each supplying organ the amount of $\mathrm{N}$ that was taken up.

4. N Reallocation. In this step N supply (N Reallocation Supply) is that coming from senescing organs. This $\mathrm{N}$ has already been taken up by the plant and converted into useful organic forms so the plant uses this in preference to mineral $\mathrm{N}$ uptake.

5. N Uptake. In this step $N$ supply of mineral $N$ from the environment (N Uptake Supply) and the arbitrator partitions its $\mathrm{N}$ uptake supply to satisfy each organs $\mathrm{N}$ demand. If $\mathrm{N}$ uptake supply is not sufficient to meet the organs $\mathrm{N}$ demand their $\mathrm{N}$ concentration will begin to fall. If total $\mathrm{N}$ demand is less than the uptake supply the crop will leave the surplus mineral $\mathrm{N}$ in the soil.

6. N Retranslocation. This is only invoked under $\mathrm{N}$ shortage when $\mathrm{N}$ reallocation and uptake cannot meet structural and metabolic $\mathrm{N}$ demands. In this step $\mathrm{N}$ supply is non-structural $\mathrm{N}$ in organs and the metabolic $\mathrm{N}$ in leaves. The arbitrator will first move non-structural $\mathrm{N}$ to meet the demand for metabolic and structural $\mathrm{N}$ of growing organs. If this is not enough to meet demand the arbitrator may also remove metabolic $\mathrm{N}$ from older leaf cohorts to meet the $\mathrm{N}$ demand of growing organs.

7. Actual DM allocation is determined once final $\mathrm{N}$ allocations have been set. The arbitrator determines if the $\mathrm{N}$ allocated to each organ is satisfy each organs structural and metabolic $\mathrm{N}$ demand. If not the arbitrator will constrain the DM growth of that organ and discards the surplus DM. This assumes that 
under severe $\mathrm{N}$ stress photosynthesis would be down regulated due to $\mathrm{N}$ inadequacy limiting sink strength.

8. Once all of these steps are complete the arbitrator sends arbitration results to each organ which in turn set the organs state changes. The mechanisms within each set method determine what pools and organ cohorts DM and $\mathrm{N}$ is assigned to or taken away from.

\section{MODEL PERFORMANCE}

We have assembled a wide range experimental data used for determining parameter values for the APSIM potato model. However, due to space constraints we will limit results to one trial that was conducted at Lincoln, New Zealand with Russet Burbank potatoes planted on the $21^{\text {th }}$ of October 1999 with a range of fertilizer treatments. The two treatments reported here are the nil fertilizer treatment and the treatment that had $300 \mathrm{~kg} / \mathrm{ha}$ of urea $\mathrm{N}$ broadcast to the soil on the day of planting. The high fertilizer treatment achieved a tuber yield of $19.5 \mathrm{tDM} / \mathrm{ha}$ and APSIM gave good predictions of both the final yield and the accumulation of tuber DM during the duration of the crop (Figure 2). By contrast the treatment that received no $\mathrm{N}$ fertilizer achieved a yield of 15.0 $\mathrm{tDM} /$ ha with the reduced tuber production coming in the second half of tuber bulking and the model predicting this pattern well. Although $\mathrm{N}$ shortage only reduced tuber yield by $\sim 25 \%$ the $\mathrm{N}$ content (Figure 2 ) of the nil $\mathrm{N}$ tubers $(125 \mathrm{~kg} / \mathrm{ha})$ was less than half that of the high $\mathrm{N}$ treatment $(320 \mathrm{~kg} / \mathrm{ha})$ and the model predicted this difference well. Treatment differences were evident at an early stage in leaf and stem DM and N content with stem being the most sensitive to $\mathrm{N}$ shortage. The model captured this by making tuber the highest priority for $\mathrm{N}$ followed by leaf then stem. This is why the model was able to accurately reproduce the early cessation of stem DM production and reduction in $\mathrm{N}$ content to maintain $\mathrm{N}$ supply and growth of the tubers in the nil $\mathrm{N}$ treatment (Figure 2). The model has been tested on a range of other experiments with different $\mathrm{N}$ application rates and is giving equally good performance with these tests. It has also been tested against experiments with different plant populations, sowing dates and irrigation treatments and accurately reproduces the effects of these management treatments.

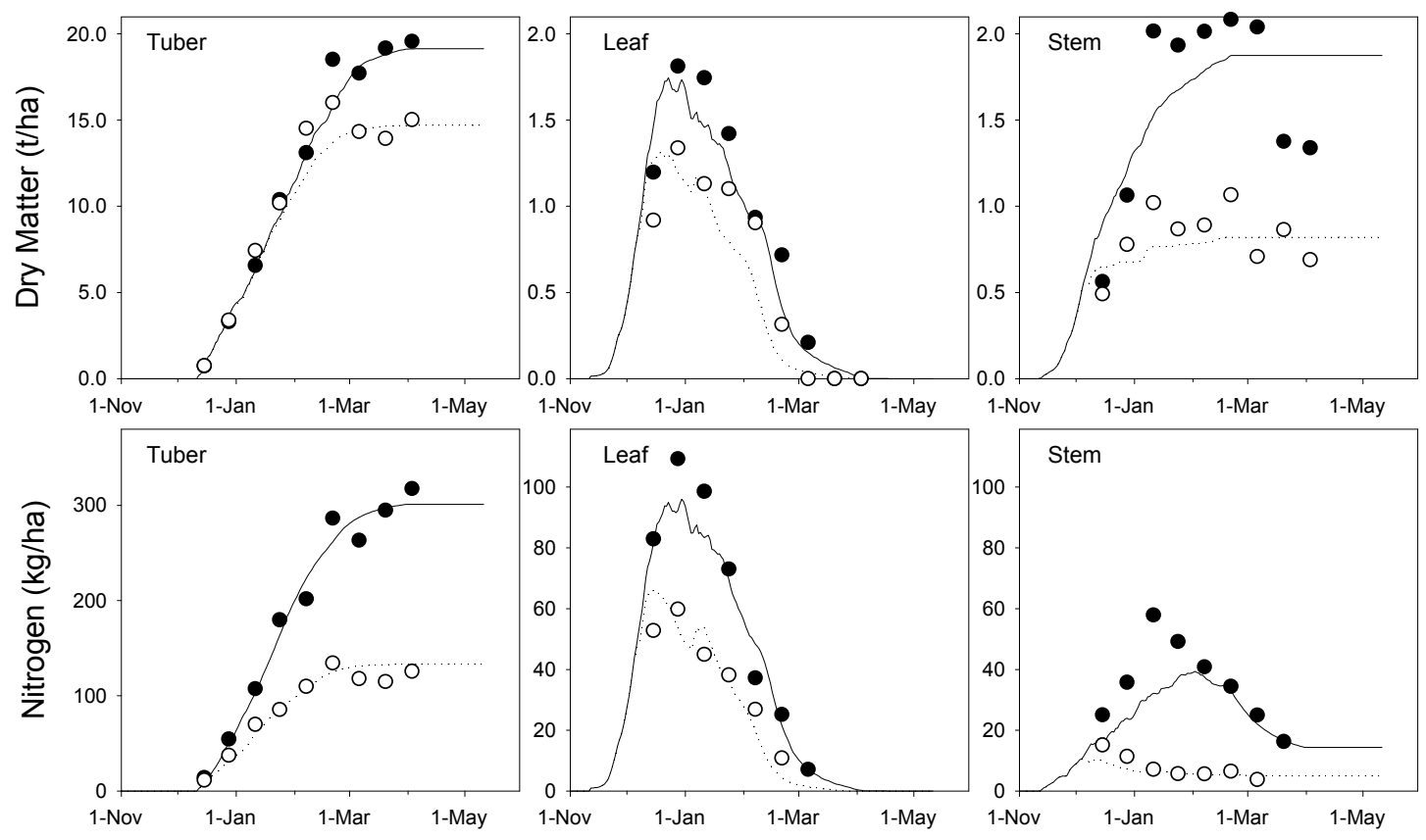

Figure 2.Time courses of observed (symbols) and predicted (lines) organ mass and nitrogen for Russet Burbank potatoes grown at Lincoln, New Zealand. Treatments received $0(\circ, \cdots \cdots)$ and $300(\bullet,-) \mathrm{kg} \mathrm{N} / \mathrm{ha}$.

\section{CONCLUSION AND FUTURE PROSPECTS}

While the APSIM potato model is still in the early stages of development, its performance is promising. It is to the best of our knowledge the most comprehensive potato model currently available. Its phytomer canopy model provides mechanisms to capture the effects of a wide range of management treatments on radiation capture, its partitioning rules providing a generic mechanism for predicting $\mathrm{N}$ responses and the APSIM infrastructure enabling comprehensive treatment of all aspects of field water and $\mathrm{N}$ balance. The APSIM user 
interface also enables quick and easy set up of various management and rotation options in simulations which will enable easy adoption of the APSIM potato model.

Beyond what is written in this paper there is a need to publish in greater detail the methodologies used to establish and parameterize the mechanisms used in the canopy and partitioning aspects of this model and a broader account of model testing. While a substantial body of experimental data has been assembled and used to test the model there is a need for more testing of the water use and water shortage response mechanisms of the model as to date they have only been checked against one experiment. There is also a need to refine phenology mechanisms to account for the effects day-length has on final leaf number and subsequent crop duration. There is also a need to parameterize the model for a wider range of cultivars (currently it is limited to Russet Burbank) and provide a method to provide reliable estimates of cultivar parameters from simple field observations. Once these outstanding issues have been addressed we will be confident the model can give accurate estimations of yield and resource use in a wide range of situations.

This model provides a good framework for building in mechanisms for the prediction of the quality of potato yields including tuber size distribution and tuber dry matter content. An element of particular importance in this respect is the effect of the treatment of seed tubers pre-planting on the behavior of the potato crop post planting. Our future plans are to add mechanisms to better quantify the dormancy phase of the crop and the development of shoots prior to planting to capture these effects. We also plan to add in a cohorting tuber model to enable the simulation of size distribution of tuber classes dependent on when they were initiated and their relative growth rates.

\section{REFERENCES}

Haverkort, A., 2007. The Canon of Potato Science: 46. Potato Crop Modelling. Potato Research, 50(3): 399402.

Holzworth, D. and Huth, N.I., 2009. Reflection + XML simplifies development of the APSIM generic PLANT model, 18th World IMACS / MODSIM Congress, Cairns, Australia.

Huth, N.I., Henderson, C. and Peake, A., 2009. Development and testing of a horticultural crop model within APSIM, 18th world IMACS / Modsim Congress, Cairns, Australia.

Jamieson, P.D., Semenov, M.A., Brooking, I.R. and Francis, G.S., 1998. Sirius: a mechanistic model of wheat response to environmental variation. European Journal of Agronomy, 8(3-4): 161-179.

Jamieson, P.D., Stone, P.J., Zyskowski, R.F., Sinton, S. and Martin, R.J., 2004. Implementation and testing of the Potato Calculator, a decision support system for nitrogen and irrigation management, Decision support systems in potato production: bringing models to practice. Wageningen Academic Publishers, Wageningen Netherlands, pp. 85-99.

Keating, B.A. et al., 2003. An overview of APSIM, a model designed for farming systems simulation. European Journal of Agronomy, 18: 267-288.

Ludwig, F. and Asseng, S., 2006. Climate change impacts on wheat production in a Mediterranean environment in Western Australia. Agricultural Systems, 90(1/3): 159-179.

Snow, V. and Huth, N., 2004. The APSIM - Micromet module. 2004/12848, HortResearch.

Struik, P., Lommen, W., Haverkort, A. and Storey, R., 2007. The Canon of Potato Science. Potato Research, 50(3): 205-206.

Vos, J., 1995. Foliar development of the potato plant and modulations by environmental factors. Modelling and parameterization of the soil-plant-atmosphere system: a comparison of potato growth models., 21-38 pp.

Wang, E., Smith, C.J., Bond, W.J. and Verburg, K., 2004. Estimations of vapour pressure deficit and crop water demand in APSIM and their implications for prediction of crop yield, water use, and deep drainage.

Australian Journal of Agricultural Research, 55(12): 1227-1240. 